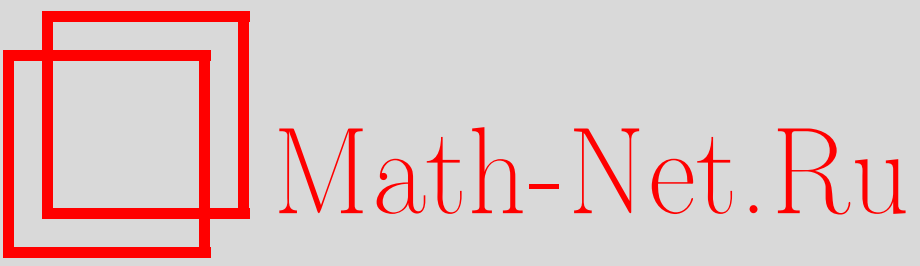

Ю. Г. Павленко, Движение спина релятивистской частицы в поле бегущей волны тока, ТМФ, 2001, том 126, номер 2, 271-282

DOI: https://doi.org/10.4213/tmf430

Использование Общероссийского математического портала Math-Net.Ru подразумевает, что вы прочитали и согласны с пользовательским соглашением

http://www.mathnet.ru/rus/agreement

Параметры загрузки:

IP: 18.234 .156 .22

26 апреля 2023 г., $16: 43: 40$ 
ТЕОРЕТИЧЕСКАЯ

И МАТЕМАТИЧЕСКАЯ

ФИЗИКА

Tом 126, № 2

февраль, 2001

(C) 2001 г.

\author{
Ю. Г. Павленко*
}

\title{
ДВИЖЕНИЕ СПИНА РЕЛЯТИВИСТСКОЙ ЧАСТИЦЫ В ПОЛЕ БЕГУШЕЙ ВОЛНЫ ТОКА
}

Уравнение Баргмана-Мишеля-Телегди представлено в гамильтоновой форме. Получено решение для заряженной частицы, движущейся в электромагнитном поле, возбуждаемом бегущей волной тока в аксиально-симметричной электродинамической системе.

\section{1. ВВЕДЕНИЕ}

В настоящее время исследования поляризационных эффектов выделились в самостоятельный раздел физики ядра и элементарных частиц. Использование поляризованных частиц дает возможность определить комбинации спиральных амплитуд, необходимые для анализа различных теоретических моделей. Для проверки применимости квантовой электродинамики в области малых передаваемых импульсов принципиальное значение имеет точное измерение аномального магнитного момента электрона $\mu^{\prime}=(g / 2-$ 1) $\mu_{0}, \mu_{0}=e \hbar / 2 m c$. Динамика поляризации влияет на формирование сфокусированного потока нейтрино, образующихся в распаде мюонов, поскольку направление вылета нейтрино определяется ориентацией спина мюона. Спиновые эффекты играют основную роль в задаче транспортировки и накопления нейтронов в магнитных ловушках.

В нерелятивистской квантовой теории анализ решений уравнения Паули приводит к наглядной физической интерпретации поляризационных явлений. Положение существенно меняется в релятивистской теории. Здесь для исследования спиновых эффектов во внешних полях необходимо вводить сохраняюшиеся ковариантные операторы поляризации [1], [2]. Движение среднего значения 4-вектора спина заряженных релятивистских частиц может быть исследовано в результате решения уравнения Баргмана-Мишеля-Телегди (БМТ) [3]. Этот подход, основанный на квазиклассическом приближении, имеет ограниченную область применимости: волновая функция частицы представляет собой локализованный пакет, движушийся по классической траектории; прирашением 4-потенциала на длинах порядка размеров пакета можно пренебречь; напряженности полей малы по сравнению с напряженностью критического поля. Следует также отметить, что в уравнении БМТ не учтена эволюция вектора спина, обусловлен-

\footnotetext{
${ }^{*}$ Специализированный учебно-научный центр им. А. Н. Колмогорова, Москва, Россия
} 
ная взаимодействием частишы с полем излучения. В частности, зависимость вероятности переходов от ориентации спина в начальном состоянии приводит к эффекту Соколова-Тернова - самополяризации при излучении релятивистских электронов, движушихся в магнитном поле [4]. В работах [5]-[7] развиты методы, позволяюшие учесть реакцию излучения на движения частицы и спина. Для создания пучка поляризованных частиц необходимы анализ деполяризуюших факторов, исследование динамики поляризации и решение проблемы управления поляризацией. В работах [8]-[10] предложен новый метод анализа орбитального и спинового движений при прохождении частищ пучка через различные элементы ускорителя. Основой этого метода, позволяюшего сушественно повысить точность численных расчетов, является операторное представление алгебры Ли.

Точные решения уравнения БМТ получены для частицы в однородном магнитном поле [11], в однородных электрическом и магнитном полях [12], [13], в поле линейно поляризованной волны [13], в суперпозиции скрешенного поля и эллиптически поляризованной волны [14], в поле циркулярно поляризованной волны, распространяющейся вдоль однородного постоянного магнитного поля [14]. В работе [15] получены точные решения уравнения БМТ в случае неоднородных полей в терминах оператора преобразования из системы покоя в лабораторную, зависящего от скорости частишы.

В настоящей работе рассмотрено движение спина заряженной релятивистской частицы в поле волны, возбуждаемой бегушей волной тока в аксиально-симметричной электродинамической системе. Показано, что решение уравнений движения частицы при произвольном способе возбуждения волны определяется решением уравнения осциллятора с переменной частотой. Получено точное решение для частишы в поле бегушей волны скачка плотности тока, представляюшего собой скачок продольной компоненты магнитного поля и вихревых поперечных электрического и магнитного полей. Уравнение БМТ преобразовано в уравнение движения 3-вектора постоянной длины, которое в результате замены переменных может быть представлено в гамильтоновой форме. Для этой цели в работе использован подход Швингера [16], [17]. В рамках теории канонических преобразований найдено точное решение уравнения БМТ при движении частицы в рассматриваемом поле бегущей волны. Вектор поляризации частицы представлен в виде преобразования поворота вектора начального состояния.

\section{2. ЭЛЕКТРОМАГНИТНОЕ ПОЛЕ ЭЛЕКТРОДИНАМИЧЕСКОЙ СИСТЕМЫ}

4-потенциал электромагнитного поля представим в виде [18]

$$
A^{\mu}(x)=\frac{1}{2}\left[\left(e_{(2)} x\right) e_{(1)}^{\mu}-\left(e_{(1)} x\right) e_{(2)}^{\mu}\right] B(k x),
$$

где $x^{\mu}=(c t, x, y, z), e_{(1)}^{\mu}=(0,1,0,0), e_{(2)}^{\mu}=(0,0,1,0), k^{\mu}=(\omega / c) n^{\mu}, n^{\mu}=(1,0,0,1)$, $k x=\omega t-\omega z / c$. Здесь использована метрика $g^{\mu \nu}=\operatorname{diag}(1,-1,-1,-1) ;$ скалярное произведение 4-векторов $a b=a^{\mu} b_{\mu}=a_{0} b_{0}-\mathbf{a b}, a^{2} \equiv a^{\mu} a_{\mu} ; B(k x)$ - произвольная функция, удовлетворяюшая естественным граничным условиям: $B(k x) \rightarrow B_{1}$ при $k x \rightarrow-\infty$, 
$B(k x) \rightarrow B_{2}$ при $k x \rightarrow+\infty$, где $B_{1}, B_{2}>B_{1}$ - постоянные величины. 4-потенциал (1) удовлетворяет волновому уравнению $\partial^{\nu} \partial_{\nu} A^{\mu}=0$ и условию Лоренца $\partial_{\mu} A^{\mu}=0$. Тензор электромагнитного поля

$$
F^{\mu \nu}=t^{\mu \nu} B(k x)+\frac{1}{2}\left[\left(e_{(1)} x\right) l^{\mu \nu}-\left(e_{(2)} x\right) f^{\mu \nu}\right] B^{\prime}
$$

где $t^{\mu \nu}=e_{(2)}^{\mu} e_{(1)}^{\nu}-e_{(1)}^{\mu} e_{(2)}^{\nu}, l^{\mu \nu}=e_{(2)}^{\mu} k^{\nu}-k^{\mu} e_{(2)}^{\nu}, f^{\mu \nu}=e_{(1)}^{\mu} k^{\nu}-k^{\mu} e_{(1)}^{\nu}, B^{\prime}=d B / d(k x)$. Напряженности электрического и магнитного полей соответственно равны $\mathbf{E}=$ $\left(y k_{0} B^{\prime} / 2,-x k_{0} B^{\prime} / 2,0\right), \mathbf{B}=\left(x k_{0} B^{\prime} / 2, y k_{0} B^{\prime} / 2, B\right), k_{0}=\omega / c$. Очевидно, $\mathbf{E B}=0$. Векторы напряженностей $\mathbf{E}$ и $\mathbf{B}$ представляют собой решения уравнения Максвелла в свободном пространстве. Для получения рассматриваемой конфигурации полей внутри цилиндрической области радиусом $\rho_{0}$ необходимо возбудить на поверхности $\rho=\rho_{0}$ ток плотностью

$$
\mathbf{j}(t, x)=\left(-\frac{y}{\rho}, \frac{x}{\rho}, 0\right) c B\left(\omega t-\frac{\omega z}{c}\right) \frac{\delta\left(\rho-\rho_{0}\right)}{4 \pi}, \quad \rho=\left(x^{2}+y^{2}\right)^{\frac{1}{2}}
$$

Отметим, что в случае $B=$ const имеем однородное постоянное магнитное поле в области $\rho<\rho_{0}$. "Бегущий" аксиальный ток индуцирует вихревые тангенциальное электрическое и радиальное магнитное поля в плоскости, перпендикулярной оси симметрии системы.

\section{3. РЕШЕНИЕ УРАВНЕНИЙ ДВИЖЕНИЯ ЧАСТИЩЫ}

Решение релятивистских уравнений движения

$$
\ddot{x}^{\mu}=\frac{e}{m c} F^{\mu \nu} \dot{x}_{\nu}
$$

будем искать в виде

$$
\dot{x}^{\mu}=-\left(e_{(1)} \dot{x}\right) e_{(1)}^{\mu}-\left(e_{(2)} \dot{x}\right) e_{(2)}^{\mu}+q_{3} n^{\mu}+q_{4} \bar{n}^{\mu}
$$

где $\bar{n}^{\mu}=(1,0,0,-1), \quad \dot{x}^{\mu}=d x^{\mu} / d \tau-4$-скорость частищы, $\dot{x}^{\mu}=\gamma(c, \mathbf{v}), \quad \gamma=$ $\left[1-\mathrm{v}^{2} / c^{2}\right]^{-1 / 2}$. Начальные данные $x^{\mu}(0)=\left(0, x_{0}, y_{0}, 0\right), \dot{x}^{\mu}(0)=u^{\mu}, u^{\mu}=\gamma_{0}\left(c, \mathbf{v}_{0}\right)$.

Образуя свертку (4) с векторами $n$ и $\bar{n}$, находим $q_{3}=\bar{n} \dot{x} / 2, q_{4}=n \dot{x} / 2$. Условие $F^{\mu \nu} n_{\nu}=0$ приводит к первому интегралу $n \dot{x}=n u$ уравнения (3). Следовательно, $k x=$ $\sigma(\tau), \sigma=k u \tau, q_{4}=n u / 2$. Поскольку $\dot{x}^{2}=c^{2}$, то $q_{3}=\left(c^{2}+\dot{x}^{2}+\dot{y}^{2}\right) / 2 n u$. Учитывая значения $q_{3}=(c \dot{t}+\dot{z}) / 2, q_{4}=(c \dot{t}-\dot{z}) / 2$, находим

$$
\begin{aligned}
& c \dot{t}=\frac{n u}{2}+\frac{1}{2 n u}\left(c^{2}+\dot{x}^{2}+\dot{y}^{2}\right)=u_{0}+\frac{1}{2 n u}\left[\dot{x}^{2}+\dot{y}^{2}-\left(u_{1}^{2}+u_{2}^{2}\right)\right], \\
& \dot{z}=-\frac{n u}{2}+\frac{1}{2 n u}\left(c^{2}+\dot{x}^{2}+\dot{y}^{2}\right)=u_{z}+\frac{1}{2 n u}\left[\dot{x}^{2}+\dot{y}^{2}-\left(u_{1}^{2}+u_{2}^{2}\right)\right] .
\end{aligned}
$$


Подставляя (4), (2) в (3), получим уравнения

$$
\begin{aligned}
& \ddot{x}-\frac{e}{m c}\left[\dot{y} B(\sigma)+\frac{y}{2} \dot{B}(\sigma)\right]=0, \\
& \ddot{y}+\frac{e}{m c}\left[\dot{x} B(\sigma)+\frac{x}{2} \dot{B}(\sigma)\right]=0 .
\end{aligned}
$$

Подстановкой

$$
x+i y=x_{+}(\tau), \quad x_{+}(\tau)=\chi \exp \left(-\frac{i \beta}{2}\right), \quad \beta(\tau)=\frac{e}{m c} \int_{-\infty}^{\tau} d \theta B[\sigma(\theta)],
$$

приведем систему (6) к уравнению осциллятора с переменной частотой (или к уравнению типа Шредингера)

$$
\ddot{\chi}+\left[\frac{e B(\sigma)}{2 m c}\right]^{2} \chi=0
$$

с начальным условием $\chi=\exp \left(-i \omega_{1} \tau / 2\right), \omega_{1}=e B_{1} / m c$ при $\tau \rightarrow-\infty$.

Пусть $w_{(1)}(\tau), w_{(2)}(\tau)$ - два линейно независимых решения уравнения (7). Тогда общее решение системы (6) имеет вид

$$
\begin{gathered}
x=\operatorname{Re} x_{+}, \quad y=\operatorname{Im} x_{+}, \\
x_{+}=\left[Q_{1} w_{(1)}+Q_{2} w_{(2)}\right] \exp \left(-\frac{i \beta}{2}\right),
\end{gathered}
$$

где $Q_{1}, Q_{2}$ - две произвольные комплексные константы. В дальнейшем нас будет интересовать решение уравнения (7) при $\tau \rightarrow \infty$, когда функция $B(\sigma)$ достигает постоянного значения $B_{2}$. В этом случае асимптотику решения можно записать в виде [19]

$$
\chi=C_{1} \exp \left(-\frac{i \omega_{2} \tau}{2}\right)+C_{2} \exp \left(\frac{i \omega_{2} \tau}{2}\right), \quad \omega_{2}=\frac{e B_{2}}{m c}
$$

Отметим, что для нахождения коэффициентов $C_{1}$ и $C_{2}$ можно использовать решения, известные в квантовой механике, поскольку асимптотики соответствуют задаче рассеяния на одномерном потенциале. Величины $1 / C_{1}$ и $C_{2} / C_{1}$ играют роли амплитуд рассеяния вперед и назад для волны, падающей на потенциал справа. Поскольку определитель Вронского пары решений не зависит от $\tau$, то из условия сохранения вронскиана $w_{(1)} \dot{w}_{(2)}-\dot{w}_{(1)} w_{(2)}$ следует соотношение $\omega_{1} / \omega_{2}=\left|C_{1}\right|^{2}-\left|C_{2}\right|^{2}$. Если $1 / p$-характерный масштаб изменения функции $B(\sigma)$, то в адиабатическом случае $|d B(\sigma) / d \tau| \ll p k u B(\sigma)$ отношение $C_{2} / C_{1} \sim \exp \left(-\pi \omega_{1} / p k u\right)$, т.е. имеет экспоненциальную малость [19]. 


\section{4. ДВИЖЕНИЕ ЧАСТИЦЫ В ПОЛЕ БЕГУЩЕГО СКАЧКА ПЛОТНОСТИ ТОКА}

Особый интерес представляет электромагнитное поле (1), для которого функция

$$
B^{2}(\sigma)=\frac{1}{2}\left(B_{1}^{2}+B_{2}^{2}\right)+\frac{1}{2}\left(B_{2}^{2}-B_{1}^{2}\right) \operatorname{th}\left[p\left(\sigma-\sigma_{0}\right)\right]+\left[\frac{1}{2} B_{0} \operatorname{ch}\left[p\left(\sigma-\sigma_{0}\right)\right]\right]^{2}
$$

принимает предельные значения $B_{1}^{2}, B_{2}^{2}$ и имеет максимум при $B_{0}^{2}>B_{2}^{2}-B_{1}^{2}$. Эта функция известна в квантовой механике как потенциал Эккарта [19].

Наибольший интерес представляет случай $p k u \gg \omega_{1}$, соответствующий резкому скачку $B(\sigma)$. Полагая $B_{0}=0$, получим функцию

$$
B(\sigma) \approx \begin{cases}B_{1}, & \sigma<\sigma_{0} \\ B_{2}, & \sigma>\sigma_{0}\end{cases}
$$

Поскольку на траекториях системы $\sigma-\sigma_{0}=k u\left(\tau-\tau_{c}\right), \tau_{c}=\sigma_{0} / k u>0$, то, интегрируя уравнения (6) по малой окрестности точки $\tau=\tau_{c}$, получим граничные условия - приращения компонент скорости частицы $\Delta \dot{x}=\left(\omega_{2}-\omega_{1}\right) y\left(\tau_{c}\right) / 2, \Delta \dot{y}=-\left(\omega_{2}-\omega_{1}\right) x\left(\tau_{c}\right) / 2$. В соответствии с (9) два линейно независимых решения уравнения (7) имеют вид

$$
w=\left\{\begin{array}{cc}
\left(\frac{2}{\omega_{1}}\right)^{\frac{1}{2}} \exp \left(-\frac{i \omega_{1} \tau}{2}\right), \quad w_{(2)}=w^{*}, & \tau \leqslant \tau_{c}, \\
\left(\frac{2}{\omega_{1}}\right)^{\frac{1}{2}}\left[D \exp \left(-\frac{i \omega_{2} \tau}{2}\right)+S \exp \left(\frac{i \omega_{2} \tau}{2}\right)\right], & \tau \geqslant \tau_{c},
\end{array}\right.
$$

где

$$
\begin{array}{ll}
D=D_{0} \exp \left(-\frac{i \omega_{1} \tau_{c}}{2}+\frac{i \omega_{2} \tau_{c}}{2}\right), & D_{0}=\frac{1}{2}\left(1+\frac{\omega_{1}}{\omega_{2}}\right), \\
S=S_{0} \exp \left(-\frac{i \omega_{1} \tau_{c}}{2}-\frac{i \omega_{2} \tau_{c}}{2}\right), & S_{0}=\frac{1}{2}\left(1-\frac{\omega_{1}}{\omega_{2}}\right) .
\end{array}
$$

Пусть $x_{0}=0, y_{0}>0, \mathbf{v}_{0}=\left(\mathrm{v}_{1}, 0,0\right)$. Тогда $\dot{x}^{\mu}(0)=\left(u_{0}, u_{1}, 0,0\right), u_{1}=u_{0} \mathrm{v}_{1}, \beta=$ $\omega_{1} \tau-\beta_{0}$. Подставляя (10) в (8) и полагая $\tau=0$, находим

$$
\left(\frac{2}{\omega_{1}}\right)^{\frac{1}{2}} Q_{1} \exp \left(\frac{\beta_{0}}{2}\right)=i R, \quad\left(\frac{2}{\omega_{1}}\right)^{\frac{1}{2}} Q_{2} \exp \left(\frac{\beta_{0}}{2}\right)=i\left(y_{0}-R\right), \quad R=\frac{u_{1}}{\omega_{1}} .
$$

В интервале $0 \leqslant \tau \leqslant \tau_{c}$ уравнения траектории имеют вид $x(\tau)=R \sin \omega_{1} \tau, y(\tau)=$ $R \cos \omega_{1} \tau+\left(y_{0}-R\right), z(\tau)=0$. Частица движется по окружности радиуса $R$, координаты центра $\left(0, y_{0}-R, 0\right)$. После подстановки соотношения $\beta=\omega_{2}\left(\tau-\tau_{c}\right)+\omega_{1} \tau_{c}-\beta_{0}$ и (10) в (8) получим решение уравнений (6) в области $\tau \geqslant \tau_{c}$ :

$$
\begin{gathered}
x(\tau)=\operatorname{Re} x_{+}, \quad y(\tau)=\operatorname{Im} x_{+} \\
x_{+}(\tau)=i\left[D_{0} R \exp \left(-i \omega_{1} \tau_{c}\right)+S_{0}\left(y_{0}-R\right)\right] \exp \left[-i \omega_{2}\left(\tau-\tau_{c}\right)\right]+ \\
+i\left[S_{0} R \exp \left(-i \omega_{1} \tau_{c}\right)+D_{0}\left(y_{0}-R\right)\right] .
\end{gathered}
$$


Полагая $\left[D_{0} R \exp \left(-i \omega_{1} \tau_{c}\right)+S_{0}\left(y_{0}-R\right)\right]=R_{2} \exp (-i \eta)$, получим компоненты 4-скорости

$$
\dot{x}(\tau)=u_{2} \cos \left[\omega_{2}\left(\tau-\tau_{c}\right)+\eta\right], \quad \dot{y}(\tau)=-u_{2} \sin \left[\omega_{2}\left(\tau-\tau_{c}\right)+\eta\right],
$$

где $u_{2}=\omega_{2} R_{2}$. Из формул (5) находим значения кинетической энергии $T=m c^{2} \dot{t}-m c^{2}$ и продольного импульса $p_{z}=m \dot{z}$ :

$$
c \dot{t}=u_{0}+\frac{u_{2}^{2}-u_{1}^{2}}{2 u_{0}}, \quad \dot{z}=\frac{u_{2}^{2}-u_{1}^{2}}{2 u_{0}},
$$

$z$-компонента трехмерной скорости $\mathrm{v}_{z}=\left(u_{2}^{2}-u_{1}^{2}\right) /\left[2 c+\left(u_{2}^{2}+u_{1}^{2}\right) / c\right]$. Частица движется по винтовой линии радиуса $R_{2}$, ось которой находится на расстоянии $r_{2}=$ $\left|S_{0} R \exp \left(-i \omega_{1} \tau_{c}\right)+D_{0}\left(y_{0}-R\right)\right|$ от оси $z$. Отметим, что в случае $\mathrm{v}_{1}=0$ имеем

$$
\dot{x}(\tau)=u_{2} \cos \omega_{2}\left(\tau-\tau_{c}\right), \quad \dot{y}(\tau)=-u_{2} \sin \omega_{2}\left(\tau-\tau_{c}\right), \quad R_{2}=S_{0} y_{0}, \quad r_{2}=D_{0} y_{0} .
$$

В другом частном случае $y_{0}=R$ находим

$$
\begin{gathered}
\dot{x}(\tau)=u_{2} \cos \left[\omega_{2}\left(\tau-\tau_{c}\right)+\omega_{1} \tau_{c}\right], \\
\dot{y}(\tau)=-u_{2} \sin \left[\omega_{2}\left(\tau-\tau_{c}\right)+\omega_{1} \tau_{c}\right], \quad R_{2}=D_{0} R, \quad r_{2}=S_{0} R .
\end{gathered}
$$

Пусть $u_{2} \gg u_{1}, u_{1} \ll c$. Тогда $\dot{t} \approx 1+(1 / 2)\left(u_{2} / c\right)^{2}, \dot{z} \approx u_{2}^{2} / 2 c$, кинетическая энергия частицы $T \approx m u_{2}^{2} / 2$, продольная и поперечная компоненты трехмерной скорости частицы $\mathrm{v}_{z} \approx\left(u_{2}^{2} / c\right)\left[2+\left(u_{2} / c\right)^{2}\right]^{-1},\left(\mathrm{v}_{x}^{2}+\mathrm{v}_{y}^{2}\right)^{1 / 2} \approx 2 u_{2}\left[2+\left(u_{2} / c\right)^{2}\right]^{-1}$. После прохождения скачка магнитного поля $z$-компонента скорости и энергия частицы возрастают.

Отметим, что при движении частишы в аксиально-симметричном неоднородном поле, задаваемом 4-потенциалом типа (1)

$$
\begin{aligned}
A^{\mu}(x) & =\left[\left(e_{(2)} x\right) e_{(1)}^{\mu}-\left(e_{(1)} x\right) e_{(2)}^{\mu}\right] G, \\
G(\rho, k x) & =\frac{1}{\rho^{2}} \int_{0}^{\rho} d \rho^{\prime} \rho^{\prime} B\left(\rho^{\prime}, k x\right), \quad \rho=\left(x^{2}+y^{2}\right)^{\frac{1}{2}},
\end{aligned}
$$

появляется возможность реализации "бетатронно-волнового" механизма ускорения. Действительно, в бетатроне $B(\rho, \omega t)$ - убываюшая функция $\rho$, которая соответствует полю, обеспечиваюшему радиальную фокусировку частиц. Функция $G(R, \sigma)$ связана с полным потоком магнитной индукции через плошадь, ограниченную окружностью радиуса $R$, соотношением $\Phi(\sigma)=2 \pi R^{2} G(R, \sigma)$. Если выполняется аналог "бетатронного правила" $\Phi\left(\sigma_{m}\right)=2 \pi R^{2} B\left(R, \sigma_{m}\right), \sigma_{m}=\omega \tau_{m}$, то в цикле ускорения $\left(0, \tau_{m}\right)$ частиша движется по цилиндрической поверхности постоянного радиуса. Кинетическая энергия в конце цикла ускорения $T_{m}=T\left(\tau_{m}\right), T_{m}=\left(e R B_{m}\right)^{2} / 2 m c^{2}$, или $T_{m}($ ЭэВ $)=$ $45\left[R(\mathrm{м}) B_{m}(\mathrm{~T} \pi)\right]^{2} / 2 m c^{2}(\mathrm{MэB})$. Отметим, что в случае обычного бетатронного ускорения кинетическая энергия $T_{m}=\left[\left(m c^{2}\right)^{2}+\left(e B_{m} R\right)^{2}\right]^{1 / 2}-m c^{2}$, или $T_{m}(\mathrm{MэB}) \approx$ $300 R($ м $) B_{m}$ (Тл).

В другом важном случае в 4-потенциале (1) функция $B(k x)$ имеет вид $B(k x)=B_{0}+$ $b \cos k x$. Тогда уравнение (7) представляет собой уравнение Хилла. Анализ областей устойчивости решений приводит к выводу о возможности реализации ускорения частиц в режиме параметрического резонанса и сепарации частиц по удельному заряду. 


\section{5. ГАМИЛЬТОНОВА ФОРМА УРАВНЕНИЯ ДВИЖЕНИЯ СПИНА}

Решение уравнения БМТ [20]

$$
\dot{a}^{\mu}=\left(\frac{g e}{2 m c}\right) F^{\mu \nu} a_{\nu}+\left(\frac{g}{2}-1\right) \frac{\dot{x}^{\mu}(a \ddot{x})}{c^{2}},
$$

удовлетворяющее условию $a \dot{x}=0$, будем искать в виде

$$
a^{\mu}=f_{1} E_{(1)}^{\mu}+f_{2} E_{(2)}^{\mu}+f_{3} E_{(3)}^{\mu}
$$

где

$$
E_{(1)}^{\mu}=e_{(1)}^{\mu}-\frac{\left(e_{(1)} \dot{x}\right) n^{\mu}}{n u}, \quad E_{(2)}^{\mu}=e_{(2)}^{\mu}-\frac{\left(e_{(2)} \dot{x}\right) n^{\mu}}{n u}, \quad E_{(3)}^{\mu}=\frac{c n^{\mu}}{n u}-\frac{\dot{x}^{\mu}}{c} .
$$

Поскольку $E_{(i)}^{\mu} E_{(k) \mu}=-\delta_{i k}$, то из условия $a^{2}=-1$ следует соотношение $\mathbf{f}^{2}=1$. Подставляя (14) в (13) и учитывая (4), получим систему уравнений

$$
\frac{d \mathbf{f}}{d \tau}=\mathbf{\Omega} \times \mathbf{f},
$$

где

$$
\boldsymbol{\Omega}=\left[\left(\frac{g}{2}-1\right) \frac{\ddot{y}}{c},-\left(\frac{g}{2}-1\right) \frac{\ddot{x}}{c},-\frac{g e B}{2 m c}\right],
$$

с начальными условиями $f_{k}(0)=-a(0) E_{(k)}(0)$. Следует отметить, что в случае движения частицы в поле, векторы напряженности которого удовлетворяют уравнениям Максвелла, всегда можно найти 4-векторы $E_{(k)}^{\mu}$, реализующие преобразование уравнения (13) в уравнение (15).

4 -вектор $a^{\mu}=\varepsilon^{\mu \nu \alpha \beta} S_{\nu \alpha} \dot{x}_{\beta} /$ с представляет собой среднее значение вектора Широкова-Паули-Любанского. Компоненты среднего значения вектора спина $\mathbf{s}$ являются пространственными компонентами антисимметричного тензора $S^{\mu \nu}=-(1 / 2 c) \varepsilon^{\mu \nu \alpha \beta} a_{\alpha} \dot{x}_{\beta}$ : $2 \mathbf{s}=\left(\mathbf{a} \dot{t}-a_{0} \dot{\mathbf{x}} / c\right), \quad S^{0 i}=-(1 / 2 c)[\mathbf{a} \times \dot{\mathbf{x}}]_{i}[20]$. В системе покоя частицы компоненты 4 -вектора $a^{\mu}=(0, \boldsymbol{\xi})$ определяются преобразованием Лоренца

$$
\begin{gathered}
0=a_{0} c \dot{t}-\mathbf{a} \dot{\mathbf{x}}, \\
\xi=\mathbf{a}-\frac{\dot{\mathbf{x}}}{c}\left[a_{0}-\frac{\mathbf{a} \dot{\mathbf{x}}}{c(1+\dot{t})}\right]=\mathbf{a}-\frac{\dot{\mathbf{x}}}{c^{2}} \frac{\mathbf{a} \dot{\mathbf{x}}}{\dot{t}(1+\dot{t})} .
\end{gathered}
$$

Скалярное произведение $2 \mathbf{s} \dot{\mathbf{x}}=\mathbf{a x} / \dot{t}=\dot{\mathbf{x}} \boldsymbol{\xi}$, характеризующее движение вектора поляризации относительно скорости, можно представить в виде

$$
\frac{\mathbf{a v}}{c}=\frac{\dot{\mathbf{x}} \boldsymbol{\xi}}{c}=\frac{c J}{n u}-\dot{t} f_{3}, \quad J=f_{3}+\frac{f_{1} \dot{x}}{c}+\frac{f_{2} \dot{y}}{c} .
$$

Отметим, что в случае $B^{\prime}(\sigma) \rightarrow 0$ величина $J=2 s_{3}$ представляет собой первый интеграл уравнений (6), (15), или в форме $c J=\left(e_{(1)} a\right)\left(e_{(1)} \dot{x}\right)+\left(e_{(2)} a\right)\left(e_{(2)} \dot{x}\right)-(n a)(\bar{n} \dot{x})-$ первый интеграл уравнений (3), (13). 
Уравнение БМТ - результат усреднения гейзенберговских уравнений движения ковариантного оператора поляризации по некогерентной смеси состояний квазиклассического волнового пакета. Поэтому оно не принадлежит к лагранжевым или гамильтоновым уравнениям и не следует из какого-либо вариационного принципа. Однако уравнение (15) можно представить в гамильтоновой форме, используя подход Швингера, установившего связь между оператором момента импульса и спаренными операторами рождения и уничтожения, которые можно ввести при рассмотрении двух гармонических осцилляторов [16], [17].

Пусть $\Psi$ - двумерный спинор-столбец с компонентами $q_{1}, q_{2}, \Psi^{\dagger}=\left(q_{1}^{*}, q_{2}^{*}\right), \sigma_{k}$-матрицы Паули $(k=1,2,3)$. Определим компоненты вектора $\mathbf{f}$ соотношениями $f_{k}=$ $(1 / 2) \Psi^{\dagger} \sigma_{k} \Psi$ :

$$
f_{1}=\frac{1}{2}\left(q_{1}^{*} q_{2}+q_{2}^{*} q_{1}\right), \quad f_{2}=\frac{i}{2}\left(q_{2}^{*} q_{1}-q_{1}^{*} q_{2}\right), \quad f_{3}=\frac{1}{2}\left(q_{1}^{*} q_{1}-q_{2}^{*} q_{2}\right) .
$$

Введем "координаты" $x_{k}=q_{k}$ и "импульсы" $p_{k}=i q_{k}^{*} \quad(k=1,2)$ с фундаментальной скобкой Пуассона $\left[x_{i}, p_{k}\right]=\delta_{i k}$. Поскольку $\left[f_{i}, f_{j}\right]=\varepsilon_{i j k} f_{k}$, то уравнение (15) приобретает гамильтонову форму $d \mathbf{f} / d \tau=[\mathbf{f}, H]$ с гамильтонианом $H=\mathbf{f} \Omega[21]$. В терминах новых переменных

$$
H=\frac{1}{2}\left[q_{1}^{*} q_{2} \Omega_{-}+q_{2}^{*} q_{1} \Omega_{+}+\left(q_{1}^{*} q_{1}-q_{2}^{*} q_{2}\right) \Omega_{3}\right]
$$

где $\Omega_{ \pm}=\Omega_{1} \pm i \Omega_{2}, \Omega_{+}=-i(g / 2-1) \ddot{x}_{+} / c$. Канонические переменные удовлетворяют уравнениям

$$
\frac{d q_{k}}{d \tau}=\left[q_{k}, H\right]
$$

или

$$
\frac{d q_{1}}{d \tau}=-\frac{i}{2}\left(\Omega_{3} q_{1}+\Omega_{-} q_{2}\right), \quad \frac{d q_{2}}{d \tau}=-\frac{i}{2}\left(\Omega_{+} q_{1}-\Omega_{3} q_{2}\right)
$$

Отметим, что уравнения Эйлера-Лагранжа для функционала

$$
I=\int d \tau L\left(q_{k}^{*}, q_{k}, \tau\right), \quad L\left(q_{k}^{*}, q_{k}, \tau\right)=\frac{i}{2}\left[q_{n} \frac{d q_{n}^{*}}{d \tau}-q_{n}^{*} \frac{d q_{n}}{d \tau}\right]+H\left(q_{k}^{*}, q_{k}, \tau\right)
$$

принимают форму уравнений Гамильтона. Сушественным достоинством гамильтонова формализма является возможность применения новых методов интегрирования канонических уравнений движения [21], [22]. Введение функционала позволяет использовать прямые вариационные методы Ритца и Бубнова-Галеркина для получения приближенных решений уравнения (15). 


\section{6. ДВИЖЕНИЕ СПИНА В ПОЛЕ БЕГУЩЕГО СКАЧКА ПЛОТНОСТИ ТОКА}

Найдем решение уравнения БМТ (13), используя методы теории канонических преобразований (КП). Подставляя в (19) решения уравнений движения (8), (10) в интервале $0 \leqslant \tau \leqslant \tau_{c}$, получим гамильтониан

$$
H=-\frac{\Lambda}{2}\left[q_{1}^{*} q_{2} \exp \left(i \omega_{1} \tau\right)+q_{2}^{*} q_{1} \exp \left(-i \omega_{1} \tau\right)\right]-\frac{1}{2}\left(\Lambda_{3}+\omega_{1}\right)\left(q_{1}^{*} q_{1}-q_{2}^{*} q_{2}\right),
$$

где $\Lambda=(g / 2-1) \omega_{1} u_{1} / c, \Lambda_{3}=(g / 2-1) \omega_{1}$. Произведем вначале $\mathrm{K} \Pi q_{1}=b_{1} \exp \left(i \omega_{1} \tau / 2\right)$, $q_{2}=b_{2} \exp \left(-i \omega_{1} \tau / 2\right)$, порождаемое производящей функцией, зависяшей от старых координат $x_{n}=q_{n}$ и новых импульсов $p_{n}^{\prime}=i b_{n}^{*}$ :

$$
F_{2}\left(x_{n}, p_{n}^{\prime}, \tau\right)=x_{1} p_{1}^{\prime} \exp \left(-\frac{i \omega_{1} \tau}{2}\right)+x_{2} p_{2}^{\prime} \exp \left(\frac{i \omega_{1} \tau}{2}\right)
$$

После замены переменных новый гамильтониан

$$
H^{\prime}=H+\frac{\partial F_{2}}{\partial \tau}=-\frac{\Lambda}{2}\left(b_{1}^{*} b_{2}+b_{2}^{*} b_{1}\right)-\frac{\Lambda_{3}}{2}\left(b_{1}^{*} b_{1}-b_{2}^{*} b_{2}\right)
$$

не зависит явным образом от собственного времени. Последуюшее КП $b \rightarrow c$ :

$$
\begin{gathered}
b_{1}=c_{1} \cos \frac{\theta}{2}-c_{2} \sin \frac{\theta}{2}, \quad b_{2}=c_{1} \sin \frac{\theta}{2}+c_{2} \cos \frac{\theta}{2} \\
\cos \theta=\frac{\Lambda_{3}}{\Omega}, \quad \sin \theta=\frac{\Lambda}{\Omega}, \quad \Omega=\left|\frac{g}{2}-1\right| \omega_{1} u_{0}, \quad u_{0}=\left[1+\left(\frac{u_{1}}{c}\right)^{2}\right]^{\frac{1}{2}},
\end{gathered}
$$

приводит гамильтониан $H^{\prime}$ к диагональной форме:

$$
h=-\frac{\Omega}{2}\left(\left|c_{1}\right|^{2}-\left|c_{2}\right|^{2}\right)
$$

Решение канонических уравнений, порождаемых гамильтонианом $(21)$, имеет вид

$$
c_{1}=A_{1} \exp \left(\frac{i \Omega \tau}{2}\right), \quad c_{2}=A_{2} \exp \left(-\frac{i \Omega \tau}{2}\right)
$$

где $A_{1}=(1+C)^{1 / 2} \exp (i \alpha / 2), A_{2}=(1-C)^{1 / 2} \exp (-i \alpha / 2), C$ и $\alpha$ - постоянные интегрирования.

Переходя в (18) к новым переменным $q \rightarrow c$, получим в области $0 \leqslant \tau \leqslant \tau_{c}$ решение уравнения (15), которое запишем в виде

$$
\begin{aligned}
& f_{1}=\left(f_{1}^{\prime} \cos \theta+f_{3}^{\prime} \sin \theta\right) \cos \varphi-f_{2}^{\prime} \sin \varphi \\
& f_{2}=\left(f_{1}^{\prime} \cos \theta+f_{3}^{\prime} \sin \theta\right) \sin \varphi+f_{2}^{\prime} \cos \varphi \\
& f_{3}=-f_{1}^{\prime} \sin \theta+f_{3}^{\prime} \cos \theta
\end{aligned}
$$


где $\varphi=-\omega_{1} \tau$,

$$
f_{1}^{\prime}=\left(1-C^{2}\right)^{\frac{1}{2}} \cos (\Omega \tau+\alpha), \quad f_{2}^{\prime}=-\left(1-C^{2}\right)^{\frac{1}{2}} \sin (\Omega \tau+\alpha), \quad f_{3}^{\prime}=C .
$$

Отметим, что КП $q \rightarrow c$ реализует переход к новому базису $\mathbf{n}_{k} \rightarrow \mathbf{n}_{k}^{\prime} \quad(k=1,2,3)$, в котором угловая скорость врашения вектора $\mathbf{f}=f_{i}^{\prime} \mathbf{n}_{i}^{\prime}$ направлена по орту $\mathbf{n}_{3}^{\prime}$. Действительно, пусть $A_{i k}(\tau)$ - матрица поворота вокруг осей 323 на эйлеровы углы $\varphi, \theta, \psi=0$, представляющие собой обычные сферические углы оси $z^{\prime}$. Тогда компоненты угловой скорости в новом базисе имеют вид $\omega_{1}^{\prime}=-\dot{\varphi} \sin \theta, \omega_{2}^{\prime}=\dot{\theta}, \omega_{3}^{\prime}=\dot{\varphi} \cos \theta$, а компоненты вектора $f$ суть $f_{i}^{\prime}=A_{i k} f_{k}$. Подставляя $f_{m}=f_{n}^{\prime} A_{n m}$ в (15) и учитьвая соотношение $d A_{n m} / d \tau=\varepsilon_{n s k} A_{s m} \omega_{k}^{\prime}$, получим уравнение [14]

$$
\frac{d f_{i}^{\prime}}{d \tau}=\varepsilon_{i k n} \Omega_{k}^{\prime} f_{n}^{\prime}
$$

Компоненты $\Omega_{k}^{\prime}=A_{k s} \Omega_{s}-\omega_{k}^{\prime}$ равны

$$
\begin{aligned}
& \Omega_{1}^{\prime}=\left(\Omega_{1} \cos \varphi+\Omega_{2} \sin \varphi\right) \cos \theta-\left(\Omega_{3}-\dot{\varphi}\right) \sin \theta, \\
& \Omega_{2}^{\prime}=-\Omega_{1} \sin \varphi+\Omega_{2} \cos \varphi+\dot{\theta}, \\
& \Omega_{3}^{\prime}=\left(\Omega_{1} \cos \varphi+\Omega_{2} \sin \varphi\right) \sin \theta+\left(\Omega_{3}-\dot{\varphi}\right) \cos \theta .
\end{aligned}
$$

Полагая $\Omega_{1}=-\Lambda \cos \omega_{1} \tau, \Omega_{2}=\Lambda \sin \omega_{1} \tau, \Omega_{3}=-\Lambda_{3}, \varphi=-\omega_{1} \tau$, находим $\Omega_{1}^{\prime}=0$, $\Omega_{2}^{\prime}=0, \Omega_{3}^{\prime}=-\Omega$. Следовательно, компоненты вектора (24) представляют собой решение уравнений (25), компоненты вектора (23) определяются матрицей поворота $f_{m}=$ $f_{n}^{\prime} A_{n m}$ и удовлетворяют уравнению (15).

Предположим, что $g>2$. Тогда $\sin \theta=u_{1} / u_{0}=\mathrm{v}_{1} / c, \cos \theta=1 / u_{0}=\left[1-\left(\mathrm{v}_{1} / c\right)^{2}\right]^{1 / 2}$. Подставляя (23) в (14), получим компоненты вектора а в области $0 \leqslant \tau \leqslant \tau_{c}$ :

$$
a_{1}=f_{1}^{\prime} u_{0} \cos \omega_{1} \tau+f_{2}^{\prime} \sin \omega_{1} \tau, \quad a_{2}=-f_{1}^{\prime} u_{0} \sin \omega_{1} \tau+f_{2}^{\prime} \cos \omega_{1} \tau, \quad a_{3}=f_{3}^{\prime} .
$$

Пусть в системе покоя $\boldsymbol{\xi}(0)=\boldsymbol{\xi}_{0}$. Из (16) найдем начальные значения компонент $a_{n}(0)=$ $a_{0 n}: a_{01}=u_{0} \xi_{01}, a_{02}=\xi_{02}, a_{03}=\xi_{03}$. Учитывая (24), (26), находим $\xi_{0 n}=f_{n}^{\prime}(0)$ и значения постоянных $C$ и $\alpha$ :

$$
\xi_{01}=\left(1-C^{2}\right)^{\frac{1}{2}} \cos \alpha, \quad \xi_{02}=-\left(1-C^{2}\right)^{\frac{1}{2}} \sin \alpha, \quad \xi_{03}=C .
$$

Подставляя (24), (27) в (26), получим решение уравнения БМТ с заданными начальными условиями. Теперь после подстановки (26) в (16) имеем

$$
\begin{gathered}
\xi_{1}=f_{1}^{\prime} \cos \omega_{1} \tau+f_{2}^{\prime} \sin \omega_{1} \tau=\xi_{01} \cos \Omega_{z} \tau+\xi_{02} \sin \Omega_{z} \tau, \\
\xi_{2}=-f_{1}^{\prime} \sin \omega_{1} \tau+f_{2}^{\prime} \cos \omega_{1} \tau=-\xi_{01} \cos \Omega_{z} \tau+\xi_{02} \sin \Omega_{z} \tau, \quad \xi_{3}=f_{3}^{\prime}=\xi_{03},
\end{gathered}
$$


где $\Omega_{z}=\Omega+\omega_{1}-$ угловая скорость прецессии вектора $\boldsymbol{\xi}$ вокруг оси $z$. В системе отсчета, врашающейся с угловой скоростью $\omega_{1}$, проекции вектора $\boldsymbol{\xi}$ на направления скорости и ускорения равны соответственно

$$
\begin{gathered}
\frac{\dot{\mathbf{x}} \boldsymbol{\xi}}{u_{1}}=f_{1}^{\prime}=\xi_{01} \cos \Omega \tau+\xi_{02} \sin \Omega \tau \\
-\left(\xi_{1} \sin \omega_{1} \tau+\xi_{2} \cos \omega_{1} \tau\right)=-f_{2}^{\prime}=\xi_{01} \sin \Omega \tau-\xi_{02} \cos \Omega \tau
\end{gathered}
$$

Эти соотношения представляют собой известный результат: проекция вектора $\boldsymbol{\xi}$ на плоскость $(x, y)$ врашается вокруг направления скорости [20].

Движение 4-вектора спина $a^{\mu}$ при $\tau \geqslant \tau_{c}$ определяется из уравнения (13) после подстановки компонент 4-скорости (11), (12). В этой области решение уравнения (25) имеет вид

$$
\begin{aligned}
& f_{1}^{\prime}=\left(1-C^{2}\right)^{\frac{1}{2}} \cos \left[\Omega_{2}\left(\tau-\tau_{c}\right)+\Omega_{1} \tau_{c}+\alpha\right], \\
& f_{2}^{\prime}=-\left(1-C^{2}\right)^{\frac{1}{2}} \sin \left[\Omega_{2}\left(\tau-\tau_{c}\right)+\Omega_{1} \tau_{c}+\alpha\right], \quad f_{3}^{\prime}=C,
\end{aligned}
$$

где $\Omega_{2}=|g / 2-1| \omega_{2} \gamma_{2}, \gamma_{2}=\left[1+\left(u_{2} / c\right)^{2}\right]^{1 / 2}$. Решение уравнения (15) следует из (23) после подстановки $(28)$ и замен $\sin \theta \rightarrow u_{2} /\left(c \gamma_{2}\right), \cos \theta \rightarrow 1 / \gamma_{2}, \varphi \rightarrow \phi=\omega_{2}\left(\tau-\tau_{c}\right)+\eta$. Подставляя $f_{k}(k=1,2,3)$ в (14), получим вектор а в области $\tau \geqslant \tau_{c}$ :

$$
\begin{gathered}
a_{1}=f_{1}^{\prime} \gamma_{2} \cos \phi+f_{2}^{\prime} \sin \phi, \quad a_{2}=-f_{1}^{\prime} \gamma_{2} \sin \phi+f_{2}^{\prime} \cos \phi, \\
a_{3}=f_{1}^{\prime}\left(\frac{u_{2} \dot{z}}{c^{2} \gamma_{2}}\right)+f_{3}^{\prime}\left(\frac{\dot{t}}{\gamma_{2}}\right) .
\end{gathered}
$$

Компоненты вектора поляризации (16) можно представить как преобразование поворота:

$$
\begin{aligned}
& \xi_{1}=\left(f_{1}^{\prime} \cos \delta-f_{3}^{\prime} \sin \delta\right) \cos \phi+f_{2}^{\prime} \sin \phi, \\
& \xi_{2}=-\left(f_{1}^{\prime} \cos \delta-f_{3}^{\prime} \sin \delta\right) \sin \phi+f_{2}^{\prime} \cos \phi, \\
& \xi_{3}=f_{1}^{\prime} \sin \delta+f_{3}^{\prime} \cos \delta,
\end{aligned}
$$

где угол $\delta$ определен соотношениями

$$
\gamma_{2} \cos \delta=1+\frac{u_{2}^{2}}{c^{2}(1+\dot{t})}, \quad \gamma_{2} \sin \delta=\frac{u_{2} \dot{z}}{c^{2}(1+\dot{t})} .
$$

Среднее значение $\langle\boldsymbol{\xi}\rangle=\left(0,0, \xi_{03} \cos \delta\right)$. Из соотношений $(30)$ следует, что в системе отсчета, врашаюшейся с угловой скоростью $\boldsymbol{\omega}=\left(0,0,-\omega_{2}\right)$, вектор $\boldsymbol{\xi}$ описывает круговой конус с радиусом основания $\left(1-\xi_{03}^{2}\right)^{1 / 2}$ с частотой врашения $\Omega_{2}$ вокруг прямой $N$, расположенной под углом $\delta$ к оси $z$ в плоскости, касательной к цилиндрической поверхности, по которой движется частища. Пусть $u_{1} \ll c$. В ультрарелятивистском случае $T \gg m c^{2}\left(u_{2} \gg c, \dot{z} \gg u_{2}\right), \cos \delta \approx 3 c / u_{2}, \sin \delta \approx\left(1-9 c^{2} / 2 u_{2}^{2}\right)$. Поскольку $\delta \approx \pi / 2$, прямая $N$ ориентирована в направлении, противоположном направлению поперечной скорости частицы.

Благодарности. Автор благодарит К. В. Степанянца за обсуждение. Работа выполнена при поддержке Российского фонда фундаментальных исследований (проект № 97-01-00957). 


\section{Список литературы}

[1] И. М. Тернов, В. Ч. Жуковский, А. В. Борисов. Квантовые процессы в сильном внешнем поле. М.: Изд-во МГУ, 1989.

[2] И. М. Тернов. Введение в физику спина релятивистских частиц. М.: Изд-во МГУ, 1997.

[3] V. Bargman, L. Michel, V. L. Telegdi. Phys. Rev. Lett. 1959. V. 2. P. 435.

[4] А. А. Соколов, И. М. Тернов. ДАН СССР. 1963. Т. 153. С. 1052.

[5] В. Н. Байер, В.М. Катков, В.С. Фадин. Излучение релятивистских электронов. М.: Атомиздат, 1973.

[6] Ю. С. Дербенев, А. М. Кондратенко. ЖЭТФ. 1973. Т. 64. С. 1918.

[7] S. Mane. Phys. Rev. A. 1987. V. 36. P. 105.

[8] K. Yokoya. Nucl. Instr. Methods. A. 1987. V. 258. P. 149.

[9] Yu. Eidelman, V. Yakimenko. Part. Acc. 1994. V. 45. № 1. P. 17.

[10] Yu. Eidelman, V. Yakimenko. Part. Acc. 1995. V. 50. № 4. P. 261.

[11] S. Chicariello. Nuovo Cimento. 1965. V. 37. P. 161.

[12] D. Zwanziger. Phys. Rev. B. 1965. V. 139. P. 1318.

[13] M. Kolsrud. Phys. Norv. 1966. V. 2. P. 51.

[14] Ю. Г. Павленко. ЯФ. 1978. Т. 28. С. 156.

[15] А. Е. Лобанов, О. С. Павлова. ТМФ. 1999. Т. 121. № 3. С. 509.

[16] J. Schwinger. On Angular Momentum. U.S. Atomic Energy Commission NYO-3071, 1952.

[17] Д. Маттис. Теория магнетизма. М.: Мир, 1967.

[18] И. А. Малкин, В. И. Манько. Динамические симметрии и когерентные состояния квантовых систем. М.: Наука, 1979.

[19] А.И. Базь, Я.Б. Зельдович, А. М. Переломов. Рассеяние, реакции и распады в нерелятивистской квантовой механике. М.: Наука, 1971.

[20] В. Б. Берестецкий, Е. М. Лифииц, Л. П. Питаевский. Квантовая электродинамика. М.: Наука, 1989.

[21] Ю. Г. Павленко. Гамильтоновы методы в электродинамике и квантовой механике. М.: Изд-во МГУ, 1988.

[22] A. М. Переломов. Интегрируемые системы классической механики и алгебры Ли. М.: Наука, 1990.

Поступила в редакцию 15.VII.2000 г., после доработки 6.IX.2000 г. 